\title{
A comparison of the apparent gloss of rendered objects presented in the lower and upper regions of the visual field
}

\author{
Hua-Chun Sun and Damien J. Mannion \\ School of Psychology, UNSW Sydney, Sydney, Australia
}

\begin{abstract}
Gloss is an aspect of surface perception that is important for understanding the material properties of the environment. Because a surface can stimulate any region of the visual field during natural viewing, it is of interest to measure the potential influence of visual field asymmetries on perceived gloss - as such asymmetries could make the perception of gloss dependent on the visual field location. Here, our aim was to compare the apparent glossiness of renderings of nondescript objects when positioned in the lower and upper regions of the visual field. In Experiment 1, participants $(n=20)$ evaluated the glossiness of objects presented simultaneously below and above central fixation. Estimates of the specular reflectance required for perceptual gloss equality indicated little effect of the visual field location. In Experiment 2, participants $(n=19)$ compared the magnitude of gloss differences across two pairs of objects in either the lower or the upper visual field. Estimates of the exponent relating specular reflectance to a gloss difference scale and a noise parameter again indicated little effect of the visual field location. Overall, these estimates are consistent with the existence of a high degree of gloss constancy across presentations in the lower and upper visual fields.
\end{abstract}

Keywords: material perception, gloss, visual field asymmetry, surface property, specular reflection

\section{Introduction}

Gloss is a surface property that is potentially informative of material characteristics and can be useful for critical tasks such as object identification (see Chadwick \& Kentridge, 2015; Fleming, 2014, for reviews). However, the majority of previous research into the human perception of gloss have involved surfaces that stimulated the centre of gaze, either due to controlled fixation or to free viewing conditions for which such central fixation is the natural behaviour. Hence, the extent to which gloss perception is affected by the visual field location that is stimulated by the surface is unclear.

Here, we concentrate on potential asymmetries in the perception of gloss for surfaces stimulating the lower and upper regions of the peripheral visual field. There have been numerous reports of asymmetries in the behavioural and neural responses for stimuli stimulating the lower and upper visual fields (reviewed in Danckert \& Goodale, 2003; Karim \& Kojima, 2010; Previc, 1990). Such asymmetries have the potential to confer differences in the perception of gloss. For example, the contrast sensitivities of the lower and upper visual

This research was funded by the Australian Government through the Australian Research Council (DP170100087 to DM).

Correspondence concerning this article should be addressed to Damien J. Mannion, School of Psychology, UNSW Sydney, NSW, Australia. Email: damien@djmannion.net fields can differ (Carrasco, Talgar, \& Cameron, 2001; Liu, Heeger, \& Carrasco, 2006; Rijsdijk, Kroon, \& van der Wildt, 1980). Given that contrast can affect perceived gloss (e.g., Marlow, Kim, \& Anderson, 2012), this asymmetry could cause the same surface to appear to have different glossiness when stimulating the lower and upper visual fields.

Differences in perceived glossiness between the vertical visual fields could also potentially arise due to functional optimisations that are more directly related to gloss. For example, the lower visual field is particularly important during walking (Marigold \& Patla, 2008; Marigold, Weerdesteyn, Patla, \& Duysens, 2007; Timmis, Bennett, \& Buckley, 2009) and glossiness is the primary visual cue that is used to judge surface slipperiness (Joh, Adolph, Campbell, \& Eppler, 2006; Lesch, Chang, \& Chang, 2008). The lower visual field is also dominant in the visual control of actions such as grasping (Danckert \& Goodale, 2003; Previc, 1990), which have also been proposed to be guided by glossiness (Fleming, 2014; Sun, Welchman, Chang, \& Di Luca, 2016). The asymmetries in the utility of gloss estimation between the vertical field fields under these particular circumstance could confer differences in the form and fidelity of gloss representations.

Our aim in this study was to measure the difference in the apparent glossiness of a rendering of a nondescript object when it is positioned in a lower compared to an upper region of the peripheral visual field. In the first experiment, we quantified the change in specular reflectance that was required for two objects that were simultaneously presented in 
the lower and upper visual fields to be equal in glossiness. In the second experiment, we compared the difference scale that relates specular reflectance and glossiness for presentations in the lower and upper visual fields. Overall, the results of these experiments indicate a negligible effect of the vertical visual field location on glossiness.

\section{Experiment 1}

\section{Methods}

Participants. A total of 25 participants were recruited from a database of students enrolled in an introductory psychology course. Participants were compensated with course credit and were naïve to the details of the study. The participants had normal or corrected-to-normal vision (selfreported), were between 18 and 28 years old, and provided written and informed consent before taking part in the experiment. The experimental protocols were approved by the Human Research Ethics Advisory Panel in the School of Psychology, UNSW Sydney.

Apparatus. Visual stimuli were presented on a Display++ LCD monitor (Cambridge Research Systems, Kent, UK) with a spatial resolution of $1920 \times 1080$ pixels, temporal resolution of $120 \mathrm{~Hz}$, mean luminance of $60 \mathrm{~cd} / \mathrm{m}^{2}$, a linear relationship between graphics card signal and luminance, and a 10-bits per pixel output resolution. Participants viewed the monitor, in an otherwise darkened cubicle, from a distance of approximately $54 \mathrm{~cm}$ using a chinrest. The chinrest and stimulus display were adjusted for each participant to position central fixation at eye level. Stimuli were created using Blender (https://www.blender .org) and Mitsuba (version 0.6.0; https: //www . mitsuba -renderer.org/index_old.html). The experiment was conducted via Matlab (MathWorks, Natick, MA), using the Psychophysics Toolbox (Brainard, 1997; Kleiner, Brainard, \& Pelli, 2007; Pelli, 1997) and the Palamedes Toolbox (Prins \& Kingdom, 2018) extensions.

Stimuli. We created a stimulus set that contained renderings of 1,620 objects, formed from the pairwise combination of 20 shape instances and 81 surface reflectances. Each shape instance was a sphere with randomly-perturbed vertices. The surface reflectances were given by the version of the Ward bidirectional reflectance distribution function (BRDF) implemented in Mitsuba (Dür, 2006; GeislerMoroder \& Dür, 2010; Walter, 2005; Ward, 1992). This BRDF has parameters for specular reflectance $\left(\rho_{s}\right)$, diffuse reflectance $\left(\rho_{d}\right)$, and roughness $(\alpha)$. We fixed the diffuse reflectance to 0.2 and the roughness to 0.2 , and varied the specular reflectance in 0.01 increments between 0 and 0.8 (81 levels).

The scene containing the object was lit by four directional light sources, positioned at $\pm 30^{\circ}$ in azimuth and elevation from the viewing axis, and was rendered using orthographic projection and with direct illumination only. To limit the influence of a specific viewpoint and to promote the perception of gloss, each object was rendered with 30 different degrees of rotation about the vertical axis. These rotations followed a sinusoidal profile between $\pm 7.5^{\circ}$.

Following rendering, the pixel intensities of each image were normalised to the highest pixel intensity obtained across all 48,600 images (20 shape instances $\times 81$ specular reflectance levels $\times 30$ object rotations). Example images are shown in Figure 1 and an example rotation sequence is shown in Movie 1.

Design and procedure. The experiment had a repeatedmeasures design with three factors that each had two levels. These within-subjects factors were the visual field location of the standard (lower or upper visual field), the specular reflectance of the standard ( 0.15 or 0.3 ), and the task (judging the more glossy or the more matte object).

Participants completed the experiment in one session of 45 minute duration. After receiving instructions and completing practice trials, participants were presented with 8 runs of experimental trials. Each run consisted of 90 trials, composed of 20 trials from each of the 4 within-subjects conditions for a single level of the task condition (judging more glossy or more matte) and 10 'catch' trials. These 90 trials were presented in random order, causing the trials from the visual field location of the standard and the specular reflectance of the standard conditions to be interleaved within a run. The order of the active task condition was shuffled across runs.

Each trial contained the simultaneous presentation of a central fixation marker $(+)$ and an object in each of the lower and upper visual fields. The horizontal and vertical lines that comprised the fixation marker were each grey and $0.5^{\circ}$ in length. Each object was shown at approximately $5.3^{\circ}$ in diameter and at $5.8^{\circ}$ eccentricity on the vertical meridian. The objects were shown for 60 frames $(500 \mathrm{~ms})$, with a linear transparency ramp from the black background over the first and last 10 frames (83ms). During the 60 frames, the objects rotated around the vertical axis on every second frame. The shape instances were randomised on each trial, separately for the two objects. The pixel intensities of each object were scaled by separate random factors between 0.2 and 1 on each trial, to mimic different illumination strengths and to discourage the use of peak luminance as the sole correlate of glossiness. A diagram of an example frame is shown in Figure 2. Following the stimulus presentation, participants were asked to indicate the object that appeared to be more glossy or more matte (depending on the active task for the run) using the keyboard.

The specular reflectance of the comparison object on a given experimental trial was determined by an adaptive Psi staircase (Kontsevich \& Tyler, 1999). Separate staircases were initialised for each of the eight conditions at the start of the session and were resumed at the start of each subse- 


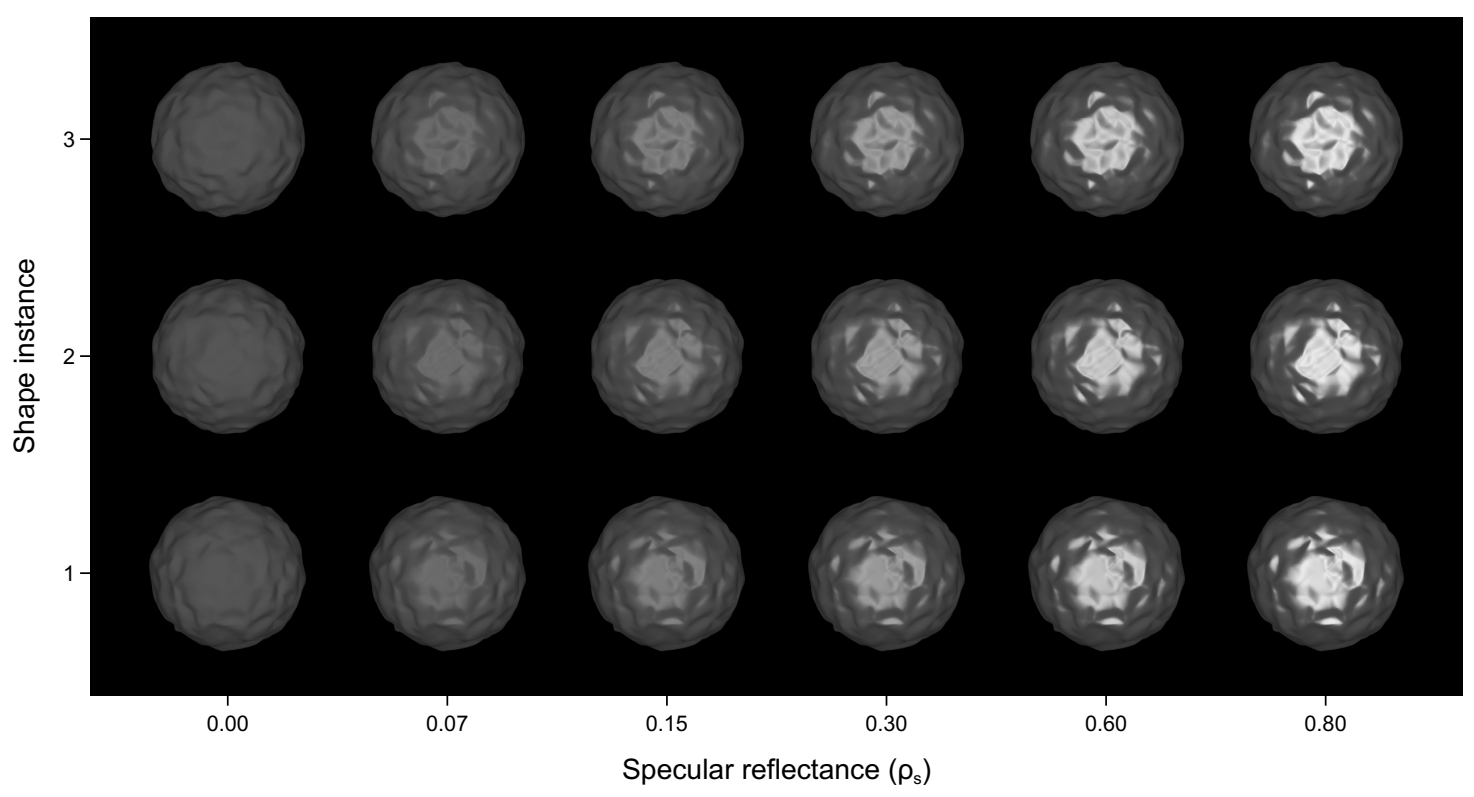

Figure 1. Examples of object renderings. The horizontal axis shows 6 (of 81) levels of specular reflectance (non-linearly spaced) and the vertical axis shows 3 (of 20) shape instances. Note that the specular reflectances of the standard object in Experiment 1 were 0.15 and 0.3 .

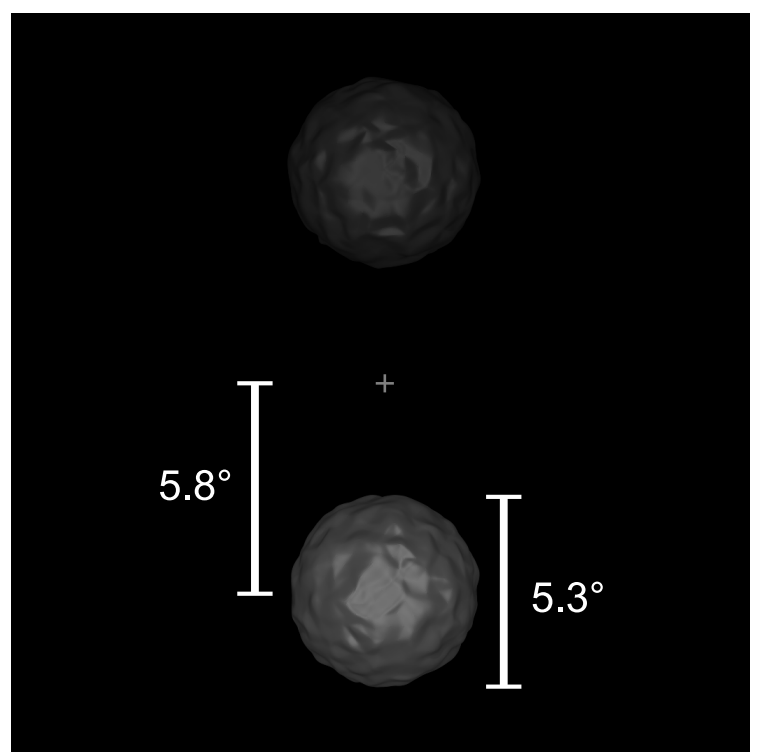

Figure 2. Diagram of an example trial frame from Experiment 1 . In this example, both objects have a specular reflectance of 0.15 and the lower and upper objects have pixel intensity modulations of 0.2 and 0.8 respectively. The text and capped lines indicate dimensions in degrees of visual angle, and were not part of the display shown to participants. quent run. On a given 'catch' trial, the standard object was randomly positioned in either the lower or upper visual field and had a specular reflectance that was randomly either 0.15 or 0.3 , and the comparison object had a specular reflectance that was randomly chosen between the five lowest and five highest levels of specular reflectance.

We included a secondary task on 10 randomly-chosen trials in each run. On such trials, the vertical line component of the fixation marker was horizontally shifted away from centre by a small amount (approximately $0.07^{\circ}$ ). Following the judgement of the more glossy or more matte object, participants were required to make an additional judgement about whether the fixation displacement was to the left or to the right. The inclusion of this secondary task was designed to encourage participants to maintain their gaze on the fixation marker, rather than on the objects, and to detect any failures to comply with this instruction (as successfully completing the task requires a spatial acuity that would be difficult to obtain if the fixation marker stimulated the periphery).

Analysis. The raw data for this experiment is available at https://doi.org/10.6084/m9.figshare .14219753 .

We first inspected the error rates on the 'catch' trials and the fixation marker task and excluded five participants due to their performance on these tasks (less than $80 \%$ accuracy on either task). The subsequent analyses were conducted with the remaining 20 participants.

The experimental trials produced a set of 12,800 (640 tri- 
als per participant $\times 20$ participants) binary values $(y)$, each indicating whether the comparison object was judged as being glossier than the standard. We consider each response as being drawn from a Bernoulli distribution in which the probability of judging the comparison object as glossier than the standard object is given by the parameter $p$ :

$$
y \sim \operatorname{Bernoulli}(p) .
$$

We assume that this probability $(p)$ can be described by a cumulative Gaussian function that is evaluated at the log of the comparison specular reflectance $(\log c)$ and with $\mu$ and $\sigma$ as parameters. For those trials where the comparison specular reflectance $(c)$ was zero, the comparison specular reflectance was set to half of the lowest non-zero level (0.005). The $\mu$ parameter captures the log specular reflectance of the comparison object when it is equiprobable for it to be perceived as more or less glossy than the standard object (the point of subjective equality; PSE), and is of primary interest. The $\sigma$ parameter captures the assumed Gaussian noise in the sensory and decision making processes and is apparent in the slope of the psychometric function. The probability of indicating that the comparison object was glossier than the standard object on a given trial could thus be given by:

$$
p=\Phi(\log c, \mu, \sigma)
$$

where $\Phi$ is the cumulative Gaussian function with mean and standard deviation as parameters. However, in recognition that our inexperienced participants are likely to have a propensity to lapse on a given trial (that is, to respond in a way that is independent of the stimulus and task), we also incorporated a lapse rate parameter $(\lambda)$ that affected the upper and lower asymptotes of the psychometric function:

$$
p=\lambda / 2+(1-\lambda) \Phi(\log c, \mu, \sigma) .
$$

The PSE parameter $(\mu)$ was given by a linear mixed model of the form:

$$
\mu=\log c+X^{\mu} \beta^{\mu}+Z^{\mu} u^{\mu} .
$$

In this formulation, $X^{\mu}$ was the fixed effects design matrix and had 12,800 rows (trials) and 7 columns (regressors) and $\beta^{\mu}$ was the set of 7 fixed effects. The first four of these fixed effects related to the key experimental manipulations and contained an intercept, a main effect of the standard object visual field location (lower or upper), a main effect of the standard specular reflectance ( 0.15 or 0.3$)$, and an interaction between the standard object visual field location and the standard object specular reflectance. The intercept regressor had a value of 1 for all trials. The main effect of the standard object visual field location regressor had a value of 1 for trials in which it was presented in the upper visual field (comparison object in the lower visual field) and -1 for trials in which it was presented in the lower visual field (comparison object in the upper visual field). The main effect of the standard object specular reflectance regressor had a value of 1 for trials in which it was 0.15 and -1 for trials in which it was 0.3 . The interaction regressor was formed from the multiplication of the two main effect regressors. While our primary interest was in estimating the $\beta^{\mu}$ value corresponding to the main effect of the standard object visual field location, visual field effects that do not produce a constant multiplicative change on the PSE (in specular reflectance units) can also induce non-zero values in the other terms.

The remaining three fixed effects were nuisance regressors that aimed to capture response biases. The first such regressor related to a potential bias for responses selecting the object presented in the upper or lower visual field (estimable via the manipulation of judging the more glossy or more matte object), and had a value of 1 on trials with standard object visual field location and task type pairings of [lower, gloss] and [upper, matte] and -1 for pairings of [lower, matte] and [upper, gloss]. The remaining two regressors related to potential biases concerning the strength of the illuminants for the two objects (which were separately randomised on each trial). The first related to a bias to responding that the object with the higher illuminant strength was glossier, and its regressor contained the log of the comparison to standard object illuminant strength ratio on each trial. The second related to a bias to responding with the object with the higher illuminant strength, independent of whether the task was to judge the more glossy or the more matte, and its regressor was formed by reversing the sign of the previous regressor on trials in which the task was to judge gloss.

In Equation $4, Z^{\mu}$ refers to the random effects design matrix. This matrix had 12,800 rows and 140 columns ( 7 fixed effects $\times 20$ participants), where each column had the value of its particular fixed effect coefficient for trials corresponding to its particular participant and a value of 0 elsewhere. The random effect values $\left(u^{\mu}\right)$ were formed from pairwise combinations of fixed effect and participant, where each participant's variation from a given fixed effect was represented as draw from a zero-mean Normal distribution with a separate standard deviation $\left(\sigma_{u}^{\mu}\right)$ for each of the fixed effects.

The log of the slope parameter $(\sigma)$ was given by a analogous linear mixed model:

$$
\log \sigma=X^{\sigma} \beta^{\sigma}+Z^{\sigma} u^{\sigma}
$$

Here, the fixed effects design matrix $\left(X^{\sigma}\right)$ had 12,800 rows and 2 columns. The first fixed effect was the intercept, and its regressor had a value of 1 for each row. The second fixed effect was the effect of the standard object specular reflectance, and its regressor had a value of -1 and 1 for trials where the standard object had specular reflectances of 0.15 and 0.3 , respectively. The random effects design matrix $\left(Z^{\sigma}\right)$ and values $\left(u^{\sigma}\right)$ were specified analogously to those for the PSE. 
For the catch trial performance, we assume that the observed number of incorrect catch trials for a given participant $\left(y_{i}^{k}\right)$ can be considered as draws from a binomial distribution:

$$
y_{i}^{k} \sim \operatorname{Binomial}\left(80, \lambda_{i} / 2\right),
$$

where $i$ indexes the participant, 80 is the number of catch trials per participant, and $\lambda$ is the lapse rate. Note that the probability of an incorrect response on a given catch trial is half the lapse rate due to guessing.

Our statistical model thus involves the parameters $\beta^{\mu}$ (7 fixed effect values), $\sigma_{z}^{\mu}$ (7 random effect standard deviations), $\beta^{\sigma}$ (2 fixed effect values), $\sigma_{z}^{\sigma}$ (2 random effect standard deviations), and $\lambda$ (20 lapse rates). We used a Bayesian framework to determine the posterior probability distribution of such parameters given our observed data; see van de Schoot et al. (2021) for a general overview, Wagenmakers et al. (2018) for an overview and evaluation of this approach in a psychology context, Lee (2018) and Kuss, Jäkel, and Wichmann (2005) in relation to psychometric functions, and Wallis, Dorr, and Bex (2015) for an example application in psychophysics. As our primary goal is to estimate the values of the parameters, rather than to perform formal hypothesis testing or model comparison (see Calin-Jageman \& Cumming, 2019), reporting summaries of the posterior distributions is our primary method of communicating the outcomes of the study (Kruschke \& Liddell, 2018). We use the median as the measure of centrality and credible intervals (equal-tailed intervals, calculated using quantiles; see Makowski, BenShachar, \& Lüdecke, 2019) as the measure of uncertainty when summarising the posterior distributions.

This Bayesian framework requires specification of prior distributions for the model parameters. We adopted the strategy of providing weakly informative priors, where the informative aspect is on the approximate scale of the parameter values. When the prior is given by a Normal distribution, we use the heuristic of identifying a value where our prior belief becomes unlikely and using half that value as the standard deviation (see Dienes, 2014). We set each of the $\beta^{\mu}$ parameters to have a prior of a Normal distribution with a mean of 0 and a standard deviation of $\log (2) / 2$. The priors for the fixed effects parameters in $\beta^{\sigma}$ were given by a Normal distribution with a mean of $\log (0.25)$ and a standard deviation of $\log (4) / 2$ (intercept) and a Normal distribution with a mean of 0 and a standard deviation of $\log (2) / 2$ (effect of standard specular reflectance). The prior for each of the random effect standard deviations $\left(\sigma^{\mu}\right.$ and $\left.\sigma^{\sigma}\right)$ was given by a half-Normal distribution with a standard deviation of $\log (2) / 2$. The prior for each participant's lapse rate $\left(\lambda_{i}\right)$ was given by a Beta distribution with $[\alpha, \beta]$ parameters of $[3,20]$.

The model was implemented in PyMC3 (version 3.11.2; Salvatier, Wiecki, \& Fonnesbeck, 2016), and Markov chain Monte-Carlo (MCMC) sampling was performed using a No-
U-turn sampler (Hoffman \& Gelman, 2014). A total of 2,000 draws were used for each of 4 independent chains in the sampling process, after discarding the initial draws $(1,000)$ used in initializing the sampler. Sampling quality was assessed by evaluation of sampling diagnostics and by visual inspection of residuals (Gelman, Goegebeur, Tuerlinckx, \& Mechelen, 2000), posterior predictive distributions (Betancourt, 2020), sampling traces, autocorrelations, and sampling metrics.

\section{Results and Discussion}

In this experiment, we asked participants to compare the glossiness of objects that were simultaneously visible in lower and upper regions of their visual field. One object was designated as the standard, and had a specular reflectance of 0.15 or 0.3 , and the other object was the comparison and had a specular reflectance that varied across trials. We analysed the resulting data using a statistical model in which the key element of interest was the estimation of the factors contributing to the point of subjective equality (PSE). To check the overall descriptive adequacy of this statistical model, we drew a series of data samples from the fitted posterior (posterior predictives) and visually compared the distribution of their summaries against a summary of the observed data. As shown in Figure 3, the statistical model appears to capture the features of the observed data that are evident in this visualisation.

The PSEs were described by a linear mixed-model (on a $\log$ scale) with four effects relating to the key experimental manipulations. The estimated posterior distributions for these four parameters are shown in Figure 4. To put the effects of such estimates in the context of the experimental manipulations, we pushed the posterior samples through the fixed-effects component of the PSE equation (Equation 4) and took the antilog to provide specular reflectance units.

This showed that the PSE estimates (median and $90 \%$ credible interval) were $0.147 \quad[0.134,0.160]$ and $0.143[0.131,0.156]$ when the standard specular reflectance was 0.15 and was in the lower and upper visual field, respectively, and 0.296 [0.272,0.324] and $0.302[0.276,0.329]$ when the standard specular reflectance was 0.3 and was in the lower and upper visual field, respectively. Overall, these measurements thus indicate that any difference in object glossiness due to the vertical visual field location are likely to be very small.

Although examining individual differences was not a primary goal of this study, we note that the standard deviation of the random effect parameter associated with the main effect of the visual field location of the standard was relatively large (median of 0.215 with a $90 \%$ credible interval of $[0.166,0.293]$, in $\log$ units). To visualise the influence of the random effects, we again calculated pushforward estimates but with the incorporation of the participant effects in the PSE equation (Equation 4). As shown in Figure 5, a sub- 

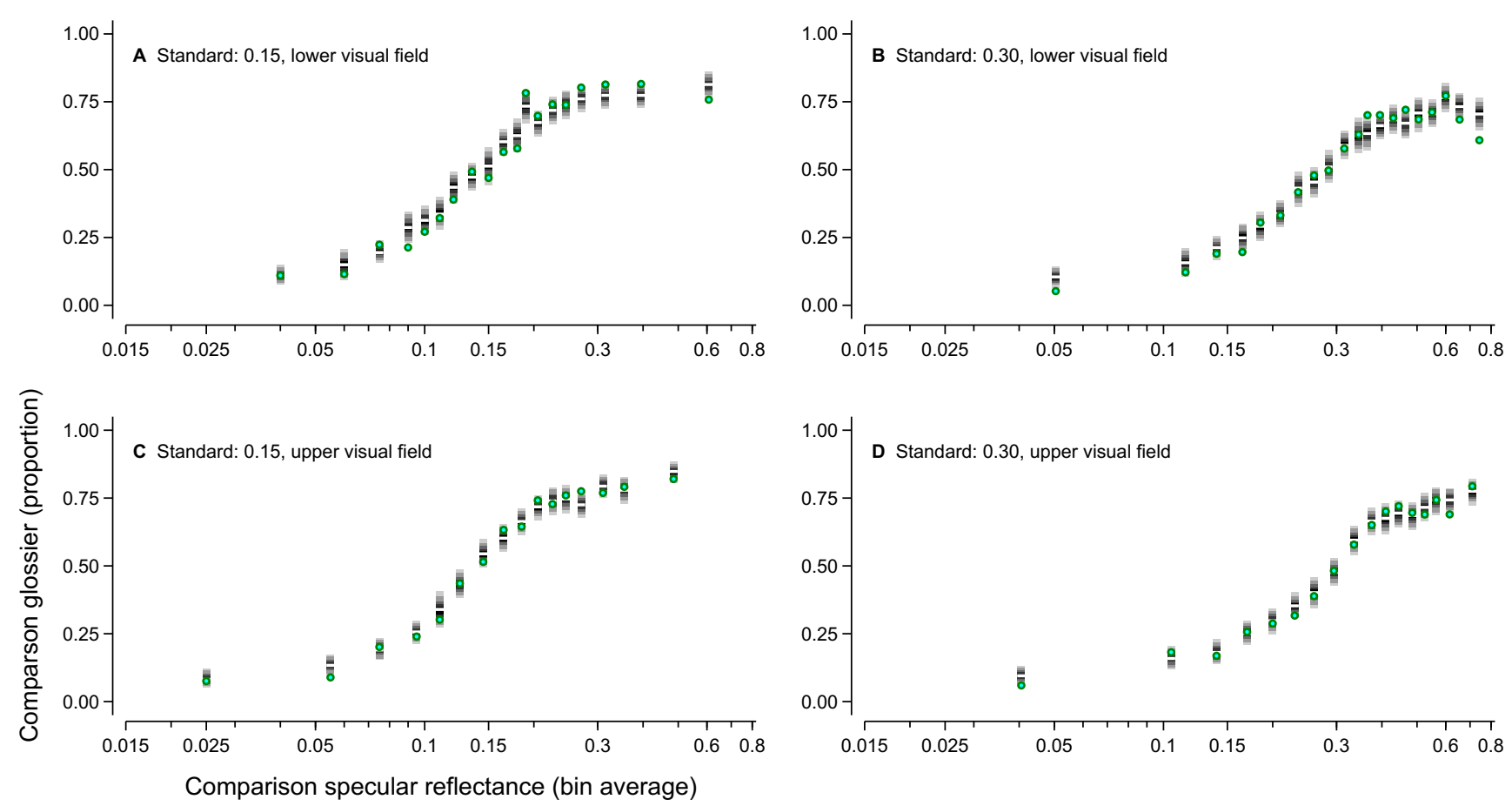

Figure 3. Summary of the observed data and posterior predictive samples, aggregated across participants, for Experiment 1. Each panel corresponds to a particular combination of the standard object specular reflectance $(0.15$ or 0.3$)$ and its presentation location (upper or lower visual field). The horizontal axis in each panel represents the mean of the comparison object specular reflectances in a bin, with 20 bins in total, and is shown with logarithmic spacing. The comparison object specular reflectances in each bin in each panel were chosen so that each bin contained an approximately equal number of trials. The vertical axis in each panel is the proportion of trials where the comparison object was judged as glossier than the standard object. The circles represent the summary of the observed data, the ribbons show credible intervals estimated from the posterior distributions (with the lightest grey representing the $90 \%$ credible interval and greys of decreasing intensity representing the $80 \%, 60 \%$, $40 \%$, and $20 \%$ credible intervals), and the white lines indicate the medians of the posterior distributions.
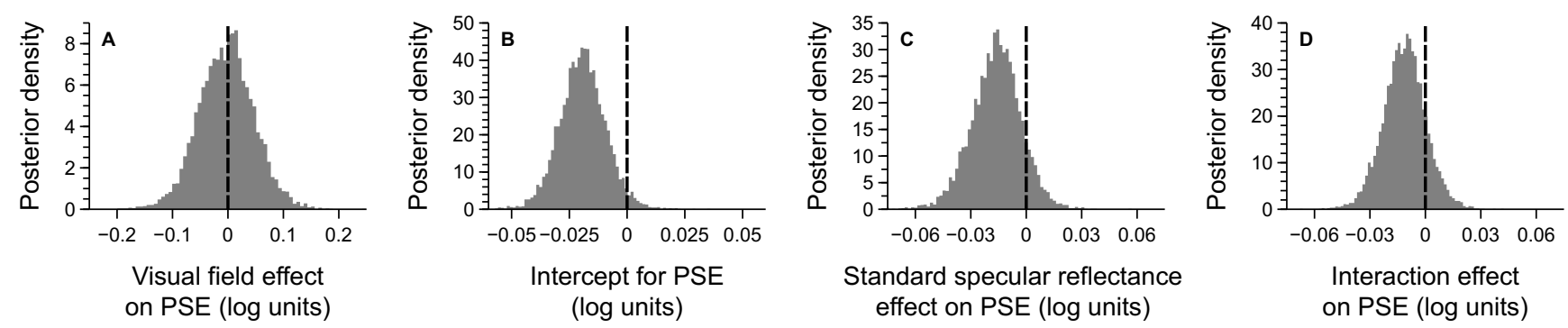

Figure 4. Summary of the posterior distributions for the key experimental parameters in Experiment 1. Each panel shows a histogram of the trace of posterior samples for a given fixed effect parameter (in $\beta^{\mu}$ ) on the PSE, with the dashed black vertical line indicating the zero point. 


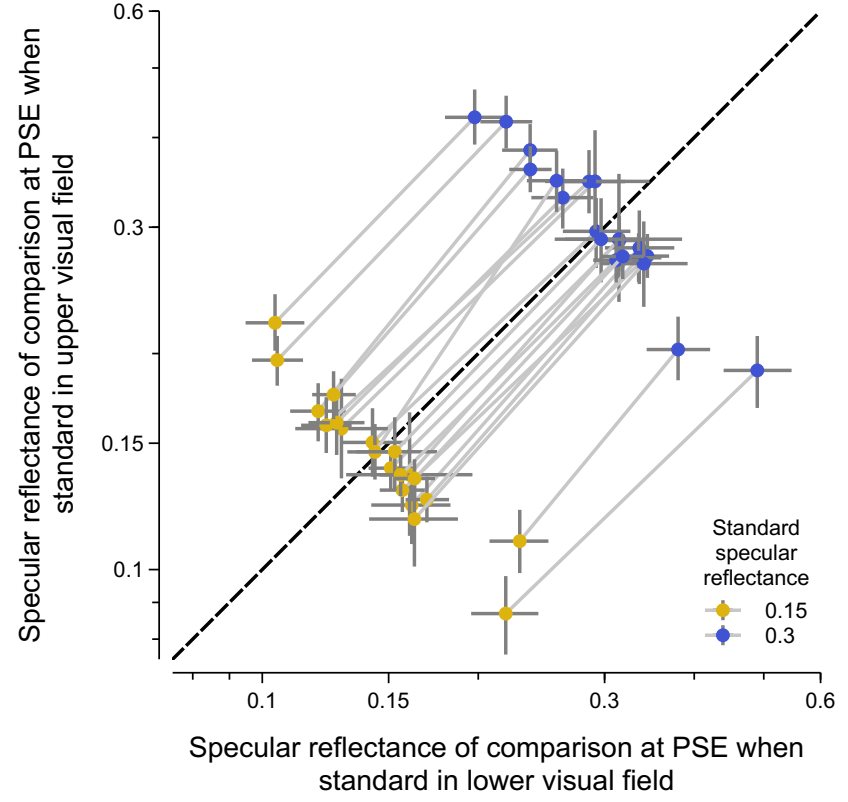

Figure 5. Participant-specific PSE estimates for Experiment 1 . The horizontal and vertical axes show the specular reflectance (with logarithmic spacing) of the comparison object when perceptually equal to a standard object presented in the lower and upper visual fields, respectively. Each point shows the median (circles) and marginal 90\% credible intervals (lines) for the posterior pushforward distribution formed from the combination of the fixed effect estimates and the estimates of a given participant's random effect. Points from the different standard specular reflectance levels are shown in different colours (gold/lighter for 0.15 and blue/darker for 0.3 ) and are joined by a grey line for points associated with a particular participant.

set of the participants showed stronger effects of the visual field location of the standard. These effects were present in both directions (i.e., both increases and decreases in the PSE when the standard was in the lower visual field) across participants, and were consistent within a participant across the specular reflectance levels of the standard.

We also note the presence of response biases in the participant behaviour. The estimated posterior distribution for the fixed-effect capturing the influence of the relative strength of the standard and comparison object illumination on glossiness responses was particularly high, with a median of -0.539 units and a $90 \%$ credible of $[-0.761,-0.302]$. This influence appeared to be primarily on the glossiness response (selecting the object with higher illumination as glossier), with a much smaller influence on the taskindependent response (selecting the object with the higher illumination as more glossy or more matte, depending on the active task; median of -0.030 with a $90 \%$ credible interval of $[-0.070,0.011])$. There was also a small response bias towards responding in favour of the object in the upper visual field, independent of the task, with its associated fixed effect posterior distribution having a median of -0.039 and a $90 \%$ credible interval of $[-0.078,0.002]$.

Overall, the results of this experiment suggest that the positioning of an object in the lower or upper visual field had little systematic effect on relative gloss judgements. Although there were some visual field differences within individuals, these were idiosyncratic and do not seem to indicate a consistent influence of a particular visual field position on apparent glossiness at the population level. To elaborate on these results and to assess their generality, we conducted a followup experiment in which we compared gloss difference scales that were estimated from presentations in the lower and upper visual fields.

\section{Experiment 2}

\section{Methods}

Participants. A total of 20 additional participants were recruited for this experiment. Recruitment details were as in Experiment 1.

Design and procedure. The experiment had a repeatedmeasures design with the visual field location of the stimuli (lower or upper visual field) as the single factor. Participants completed the experiment in a single session of 45 minute duration. After receiving instructions and completing practice trials, participants were presented with six runs of experiment trials. Each run consisted of 70 trials, composed of 30 trials from each of the 2 within-subjects conditions and 10 'catch' trials. These 70 trials were presented in random order, causing the trials from the visual field location of the stimuli condition to be interleaved within a run.

Each trial contained two temporal intervals that were each $500 \mathrm{~ms}$ in duration. During a given interval, two objects were presented in the left and right regions of either the lower or upper visual field. The objects were each shown at $6.9^{\circ}$ eccentricity and had a horizontal distance of $7.7^{\circ}$ between their centres. The objects were ramped in and out in their visibility and rotated around their vertical axes during their presentation, as in Experiment 1. Between the two intervals, there was a $400 \mathrm{~ms}$ period in which no objects were presented. The shape instances were randomised on each trial, separately for the four objects. As in Experiment 1, the pixel intensities of each object were scaled by separate random factors between 0.2 and 1 on each experimental trial. For the catch trials, the random factors were between 0.5 and 0.7 to help ensure the predictability of responses during lapses. A central fixation marker was shown throughout the trial, as in Experiment 1. Example frames from each interval are shown in Figure 6. Following the stimulus presentation, participants were asked to indicate whether which interval contained the pair of objects with a greater difference in their gloss. 


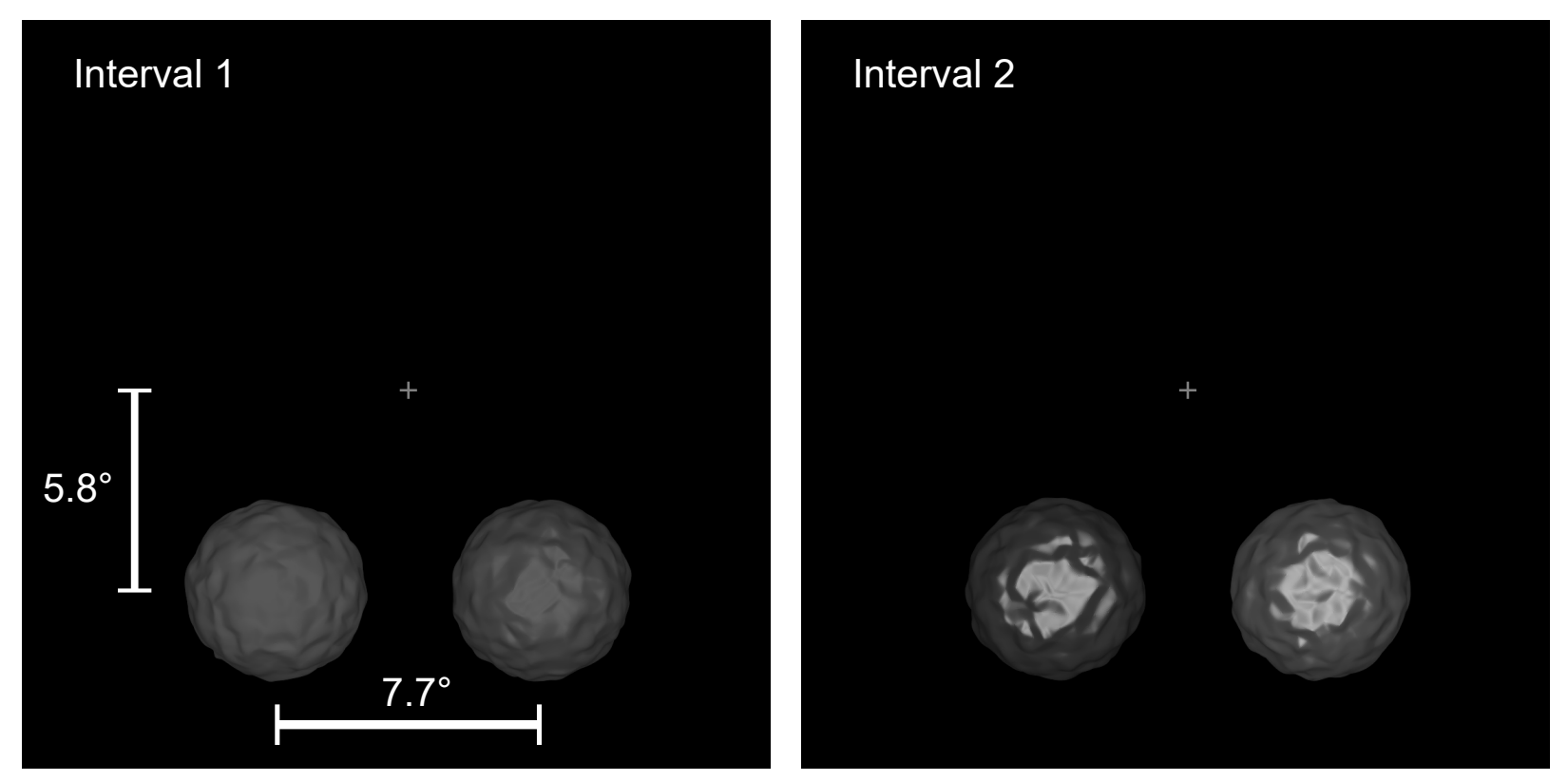

Figure 6. Diagram of example frames from a trial in Experiment 2. In this example, the first interval contains the $(a, b)$ pair (left to right) and the second interval contains the $(d, c)$ pair (left to right). The specular reflectances of $(a, b, c, d)$ are $0.01,0.07,0.47$, and 0.8 ; these were chosen to demonstrate perceptual gloss differences that would be approximately similar based on the results of the experiment. The luminance modulations of $(a, b, c, d)$ are $0.89,0.71,0.81$, and 0.41 (randomly chosen). The text and capped lines indicate dimensions in degrees of visual angle, and were not part of the display shown to participants.

The four objects on each trial had different levels of specular reflectance, represented as the quadruple $(a, b, c, d)$ in which $(a, b)$ and $(c, d)$ are the specular reflectances of the objects shown in the two temporal intervals. A set of 4,845 non-overlapping $(a<b<c<d)$ potential quadruples was formed from 20 specular reflectance levels, with 8 levels linearly spaced between 0.01 and 0.08 and 12 levels nonlinearly spaced between 0.1 and 0.8 . The quadruple shown on a given experimental trial was determined by an adaptive Psi staircase (Kontsevich \& Tyler, 1999). On a given 'catch' trial, the objects in one pair were assigned the minimum and maximum specular reflectances ( 0 and 0.8$)$ and the objects in the other pair were assigned the same (random) reflectance level. The presentation order of the pairs and the horizontal display order of the objects within a pair were randomised on each trial.

As in Experiment 1, we included a secondary task (on 10 trials of each run) to indirectly monitor the adherence to the fixation instruction. In this experiment, the horizontal arm of the fixation cross became either longer or shorter (by approximately $0.14^{\circ}$ ) during one of the stimulus intervals. This change in task, relative to Experiment 1, was designed to avoid any lateral shift of attention, given the lateral separation of the presented objects, that may have been evoked by the position task in Experiment 1. Following the judge- ment on the experimental task, participants were required to make an additional judgment about the relative direction of the length change.

Analysis. The raw data for this experiment is available at https://doi.org/10.6084/m9.figshare .14251661 .

We excluded one participant based on their error rates on the 'catch' trials and the fixation marker task (less than $80 \%$ accuracy on either task). The subsequent analyses were conducted with the remaining 19 participants.

The experimental trials produced a set of 6,840 (360 trials per participant $\times 19$ participants) binary values $(y)$, each indicating whether the perceived gloss difference between $(c, d)$ was greater than the perceived gloss difference between $(a, b)$. We consider each response as being drawn from a Bernoulli distribution in which the probability of responding $(c, d)$ is given by the parameter $p$.

According to the difference scaling method (Knoblauch \& Maloney, 2008; Maloney \& Yang, 2003), the observer calculates the difference $(\delta$ ) between the absolute differences in the perceptual difference scale representation of the pairs:

$$
\delta=|\Psi(d)-\Psi(c)|-|\Psi(b)-\Psi(a)|
$$

where $\Psi$ transduces the physical specular reflectance into the perceptual difference scale. Here, we assume that $\Psi$ has a 
parametric form (Knoblauch \& Maloney, 2008) given by a power function:

$$
\Psi\left(\rho_{s}\right)=\left(\rho_{s} / 0.8\right)^{n}
$$

in which $\rho_{s}$ is the specular reflectance, 0.8 is a normalising constant (the maximum $\rho_{s}$ ), and $n$ is an exponent. The difference value $(\delta)$ is assumed to be subject to Gaussian noise (accumulated from a variety of sources; Kingdom \& Prins, 2010) with a mean of 0 and a standard deviation of $\sigma$, giving the probability of indicating that the perceptual difference between $(c, d)$ was greater than between $(a, b)$ on a given trial as:

$$
p=1-\Phi(0, \delta, \sigma),
$$

where $\Phi$ is the cumulative Gaussian function evaluated at 0 and with mean and standard deviation as parameters. As in Experiment 1, we incorporated lapsing behaviour into the psychometric function:

$$
p=\lambda / 2+(1-\lambda)(1-\Phi(0, \delta, \sigma)) .
$$

Given that Experiment 1 showed that participants could be biased by the strength of the illumination, we incorporated consideration of such a bias by evaluating $\Phi$ at the value $k$ rather than at the constant 0 :

$$
p=\lambda / 2+(1-\lambda)(1-\Phi(k, \delta, \sigma)) .
$$

Positive values of $k$ reflect a bias towards responding that the $(c, d)$ difference was less than the $(a, b)$ difference.

The exponent parameter $(n)$ was given by a linear mixed model:

$$
\log n=X^{n} \beta^{n}+Z^{n} u^{n} .
$$

In this formulation, $X^{n}$ was the fixed effects design matrix and had 6,840 rows (trials) and 2 columns (regressors) and $\beta^{n}$ was the set of 2 fixed effects. The first fixed effect was the intercept and its regressor had a value of 1 for all trials. The second fixed effect related to the effect of the visual field location (lower or upper), and its regressor had a value of -0.5 for trials in which the objects were presented in the lower visual field and 0.5 for trials in which the objects were presented in the upper visual field. Its $\beta^{n}$ value thus represents the change, in log units, to the exponent due to the visual field location of the stimuli, and is of primary interest. The variable $Z^{n}$ refers to the random effects design matrix that had 6,840 rows (trials) and 38 columns (2 fixed effects $\times 19$ participants), and was defined analogously to previous random effects (such as Equation 4). The noise parameter $(\sigma)$ was given by a similar linear mixed model:

$$
\log \sigma=X^{\sigma} \beta^{\sigma}+Z^{\sigma} u^{\sigma},
$$

where $X^{\sigma}$ and $Z^{\sigma}$ were the same as $X^{n}$ and $Z^{n}$. The bias parameter $(k)$ was also given by a similar linear mixed model:

$$
k=X^{k} \beta^{k}+Z^{k} u^{k} .
$$

Here, the fixed effects design matrix $\left(X^{k}\right)$ had 6,840 rows (trials) and 1 column, which was given by the difference in the absolute values of the log ratios of the illumination strengths $(i)$ of the two pairs:

$$
X^{k}=\left|\log \left(i_{d} / i_{c}\right)\right|-\left|\log \left(i_{b} / i_{a}\right)\right| .
$$

The random effects matrix $\left(Z^{k}\right)$ was analogous to previous definitions. The catch trial performance was modelled as in Experiment 1.

Overall, the statistical model involved the parameters $\beta^{n}$ ( 2 fixed effect values), $\sigma^{n}$ ( 2 random effect standard deviations), $\beta^{\sigma}$ (2 fixed effect values), $\sigma^{\sigma}$ (2 random effect standard deviations), $\beta^{k}$ ( 1 fixed effect value), and $\sigma^{k}$ (1 random effect standard deviation). We used the same Bayesian framework as in Experiment 1 to estimate the posterior probability distribution of such parameters given our observed data. We set the two $\beta^{n}$ parameters to have a prior of a Normal distribution with a mean of 0 and a standard deviation of $\log (8) / 2$ (intercept) or $\log (4) / 2$ (visual field effect). The priors for the two $\beta^{\sigma}$ parameters were given by Normal distributions with a mean of $\log (0.1)$ and standard deviation of $\log (4) / 2$ (intercept) and a mean of 0 and standard deviation of $\log (4) / 2$ (visual field effect). The prior for the $\beta^{k}$ parameter was given by a Normal distribution with a mean of 0 and a standard deviation of 1 . The prior for each of the random effect standard deviations $\left(\sigma^{n}, \sigma^{\sigma}\right.$, and $\left.\sigma^{k}\right)$ was given by a half-normal distribution with a standard deviation of $\log (2) / 2$. The prior for each participant's lapse rate $\left(\lambda_{i}\right)$ was given by a Beta distribution with $[\alpha, \beta]$ parameters of $[3,20]$.

\section{Results and Discussion}

In this experiment, we asked participants to compare the relative magnitude of the differences in the gloss of two temporally-separated presentations of two objects in either the lower or upper visual fields. We used a parametric difference scaling model (Knoblauch \& Maloney, 2008; Maloney \& Yang, 2003) to estimate the effect of the visual field location on the exponent that relates specular reflectance to a gloss difference and on the noise term. As in Experiment 1, we first sought to confirm the overall descriptive adequacy of our statistical model. Unlike Experiment 1, the dimensions of such a visualisation are not as obvious. Here, each trial can vary along four stimulus dimensions that correspond to the specular reflectances of the four objects, $(a, b)$ and $(c, d)$, shown within a trial. However, the non-overlapping nature of the quadruples and the consequences of the adaptive staircase algorithm allow for dimensionality reduction. First, all trials had the lowest specular reflectance at a single value $(0.01)$. Second, nearly all trials $(98.8 \%)$ had the highest specular reflectance at a single value $(0.8)$. Finally, the specular reflectances of the intermediate values are highly (though imperfectly) correlated (Pearson's $r$ of 0.99). We can thus rea- 
sonably visualise participant responses as a function of a single stimulus dimension, which we set as analogous to the observer decision variable ( $\delta$; see Equation 7$)$ under a linear transducer: $|d-c|-|b-a|$. As shown in Figure 7, this visualisation indicates that the statistical model appears to reasonably approximate the key features of the observed data.

The difference scales were parameterised by the exponent $(n)$, which captures the form of the relationship between the physical specular reflectance and the gloss difference scale, and the noise $(\sigma)$, which captures the variability in the internal response and decision-making processes. Our primary interest was in measuring the effect of visual field position on these parameters, estimated via linear mixed models (on log scales). The estimated posterior distributions for these parameters are shown in Figure 8. To put the effects of such estimates into the context of a difference scale, we pushed the posterior samples through the fixedeffects component of the difference scale equation (Equation 7) to produce the scales shown in Figure 9. The median and $90 \%$ credible interval of the resulting exponents were $0.391[0.296,0.522]$ and $0.401[0.304,0.536]$ for the lower and upper visual fields, respectively. The form of these difference scales is similar to those obtained using related methods (Cheeseman, Ferwerda, Maile, \& Fleming, 2021; Ferwerda, Pellacini, \& Greenberg, 2001; Obein, Knoblauch, \& Viéot, 2004; Pellacini, Ferwerda, \& Greenberg, 2000; Samadzadegan, Baar, Urban, Segovia, \& Blahová, 2015), in that the difference scale has a compressive non-linearity as a function of specular reflectance. This form of difference scale is also evident in Figure 7, in that $|d-c|-|b-a|$ is positive at the point at which it is equiprobable for $|d-c|$ to be judged as less or greater than $|b-a|$.

Given the apparent individual differences that were evident in Experiment 1, we also examined the estimates of the exponent and noise parameters for lower and upper visual field presentation for each participant. We pushed the posterior distributions through the equations for the exponent (Equation 12) and noise (Equation 13), and visualise the antilogs of the resulting distributions in Figure 10. Unlike in Experiment 1, there is little indication of substantial individual differences in this experiment. A possible reason for this between-experiment discrepancy is that the individual visual field effects in Experiment 1 could have been driven by an overall change in the degree of glossiness between the lower and upper visual fields - such a change may not be evident in the difference scales that were estimated separately for the lower and upper visual fields in Experiment 2. The discrepancy may also relate to the change in the stimulus presentation approaches between the two experiments; from the simultaneous presentation of objects in the lower and upper visual fields on the vertical meridian in Experiment 1 (Figure 2) to the simultaneous presentation of objects in either the lower or upper visual field and away from the vertical meridian in Experiment 2 (Figure 6).

Similar to Experiment 1, we again find that the responses of participants were biased by the simulated illumination of the objects. The posterior distribution for the parameter capturing this influence had a median of -0.062 with a $90 \%$ credible interval of $[-0.107,-0.022]$. However, this effect is considerably smaller than the effect of the illumination on glossiness responses in Experiment 1, as shown in Figure 11.

\section{General Discussion}

In two experiments, we measured the influence of the vertical visual field position (lower or upper) on the perceived glossiness of a rendering of a nondescript object. Using both direct and difference-of-difference comparisons, we find that any effect of the vertical visual field position was likely to be very small. On the basis of these estimates, we tentatively conclude that the vertical visual field location that is stimulated by a surface has little influence on its apparent glossiness.

However, this study has a number of important limitations that caution against a broad generalisation of this conclusion. First, our stimulus and display parameters may not have been able to elicit a sufficiently vivid impression of gloss that could then be modulated by a visual field effect. We did not incorporate realistic illumination fields (Fleming, Dror, \& Adelson, 2003), a high range of luminance variation (Phillips, Ferwerda, \& Luka, 2009), stereoscopic cues (Blake \& Bülthoff, 1990; Wendt, Faul, Ekroll, \& Mausfeld, 2010), or meaningful spatial context (Adams, Kucukoglu, Landy, \& Mantiuk, 2018)—each of which could enhance the impression of gloss. Second, we only sampled a small number of locations within the vertical visual fields. Previous reports of vertical visual field asymmetries have described heterogeneities within the visual fields (Carrasco et al., 2001, for example), and our stimuli may not have stimulated such locations. Furthermore, as we did not use eye tracking, we cannot be certain that the intended visual field locations were indeed stimulated during the experiment (although we attempted to detect such instances via a task at central fixation). Finally, we noted previously that the presence of vertical visual field asymmetries may be produced by functional differences relating to tasks such as grasping and walking. However, our stimuli and tasks did not support or motivate such behaviours - the presence of which may be critical to eliciting vertical visual field asymmetries.

Despite such limitations, this study provides an initial basis from which to assess the effect of the vertical visual field location on the apparent glossiness of a rendered object. Future research may usefully be directed towards establishing a broader understanding of the constancy, or lack thereof, of the glossiness impression that arises across the stimulation of different regions of the visual field. 

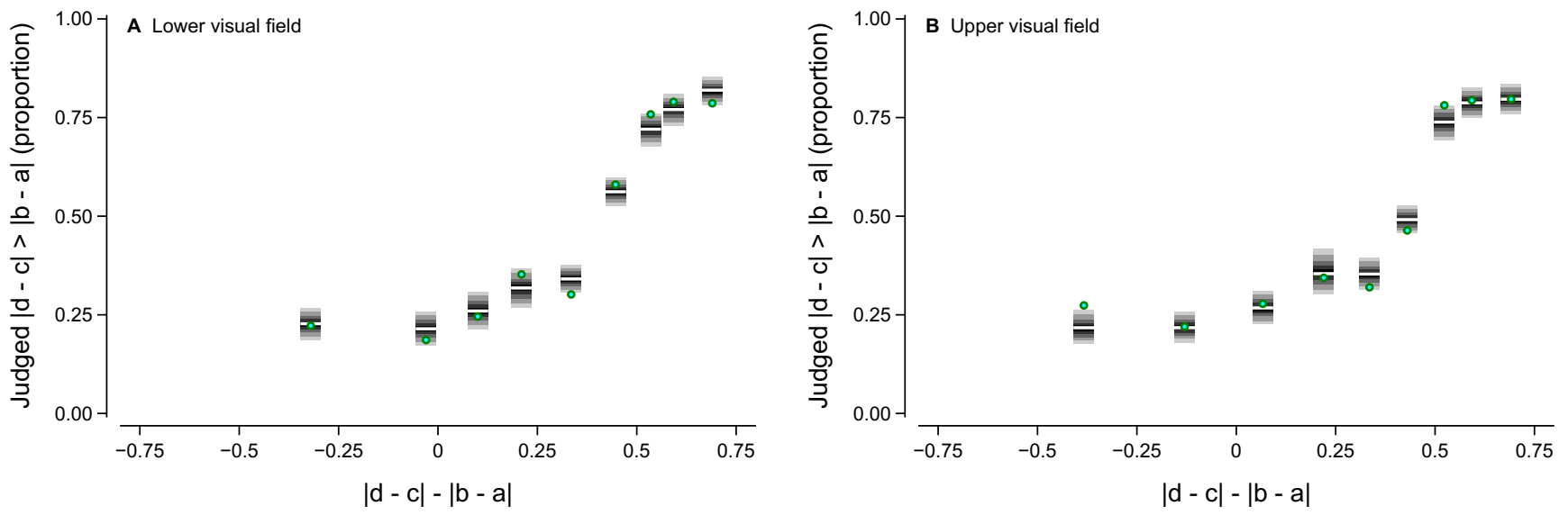

Figure 7. Summary of the observed data and posterior predictive samples, aggregated across participants, for Experiment 2. The panels depict trials where the objects were shown in the lower $(\mathbf{A})$ and upper $(\mathbf{B})$ visual field. The horizontal axis represents the mean of the difference of absolute differences calculation (where $a, b, c$, and $d$ are the specular reflectance values of the four objects shown on a given trial) in a bin, with 9 bins in total. The bins were selected so that the summary in each bin was formed from an approximately equal number of trials. The vertical axis is the proportion of trials where the absolute gloss difference of the $(c, d)$ presentation was judged as larger than that of the $(a, b)$ presentation. The figure conventions are as in Figure 3.
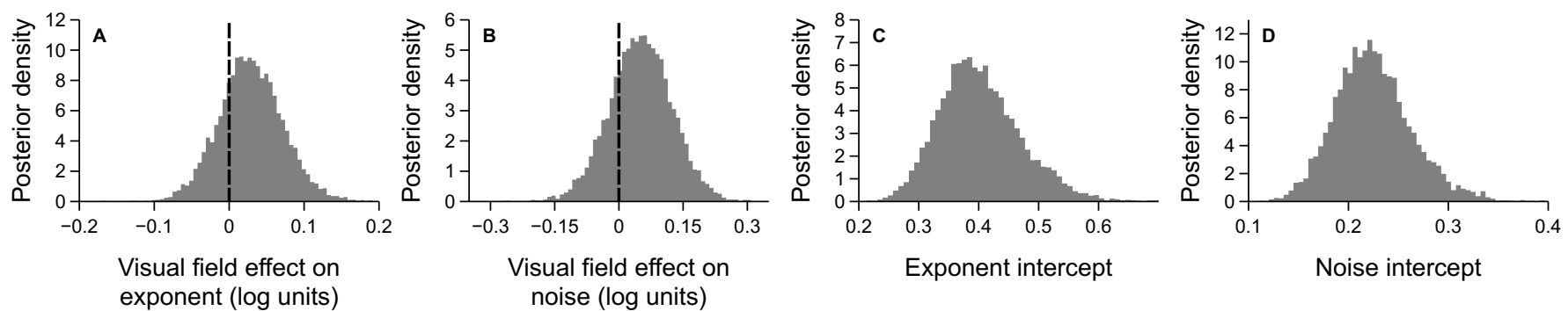

Figure 8. Summary of the posterior distributions for the key experimental parameters in Experiment 2. Each panel shows a histogram of the trace of posterior samples for a given fixed effect parameter (from $\beta^{n}$ for the exponent and from $\beta^{\sigma}$ for the noise), with the dashed black vertical line indicating the zero point.

\section{References}

Adams, W. J., Kucukoglu, G., Landy, M. S., \& Mantiuk, R. K. (2018). Naturally glossy: Gloss perception, illumination statistics, and tone mapping. Journal of Vision, 18(13), 1-16.

Betancourt, M. (2020). Towards a principled Bayesian workflow. Retrieved from https:// betanalpha.github.io/assets/case_studies/ principled_bayesian_workflow.html

Blake, A., \& Bülthoff, H. (1990). Does the brain know the physics of specular reflection? Nature, 343(6254), 165-168.

Brainard, D. H. (1997). The psychophysics toolbox. Spatial Vision, 10(4), 433-436.

Calin-Jageman, R. J., \& Cumming, G. (2019). Estimation for better inference in neuroscience. eNeuro, 6(4), 1-11.
Carrasco, M., Talgar, C., \& Cameron, E. (2001). Characterizing visual performance fields: effects of transient covert attention, spatial frequency, eccentricity, task and set size. Spatial Vision, 15(1), 61-75.

Chadwick, A., \& Kentridge, R. (2015). The perception of gloss: A review. Vision Research, 109, 221-235.

Cheeseman, J. R., Ferwerda, J. A., Maile, F. J., \& Fleming, R. W. (2021). Scaling and discriminability of perceived gloss. Journal of The Optical Society of America A-Optics, Image Science, and Vision, 38(2), 203 210.

Danckert, J. A., \& Goodale, M. A. (2003). Ups and downs in the visual control of action. In S. H. Johnson-Frey (Ed.), Taking action: Cognitive neuroscience perspectives on intentional acts (pp. 29-64). Cambridge, MA: MIT Press.

Dienes, Z. (2014). Using Bayes to get the most out of non- 


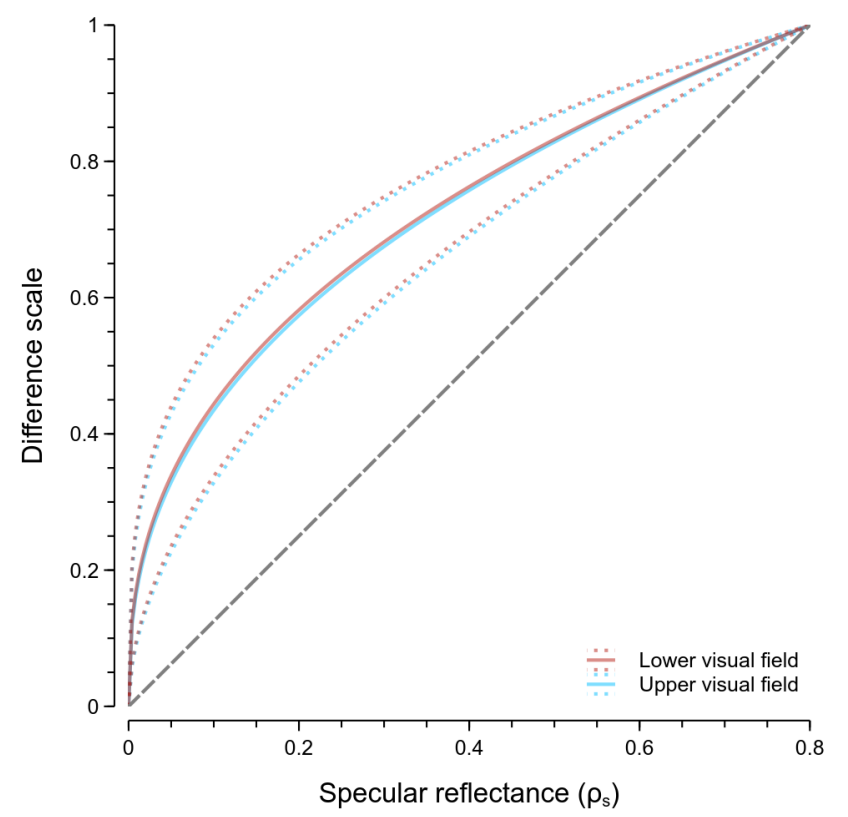

Figure 9. Summaries of the difference scales for lower and upper visual field presentation in Experiment 2. The horizontal axis shows the physical specular reflectance of the object and the vertical axis shows the estimated difference scale. The darker red and lighter blue markings denote the lower and upper visual field, respectively, with the continuous lines showing the median and the dotted lines showing the $90 \%$ credible interval.

significant results. Frontiers in Psychology, 5(781), $1-17$.

Dür, A. (2006). An improved normalization for the Ward reflectance model. Journal of Graphics Tools, 11(1), 51-59.

Ferwerda, J. A., Pellacini, F., \& Greenberg, D. P. (2001). Psychophysically based model of surface gloss perception. In Human vision and electronic imaging VI (Vol. 4299, pp. 291-301).

Fleming, R. W. (2014). Visual perception of materials and their properties. Vision Research, 94, 62-75.

Fleming, R. W., Dror, R. O., \& Adelson, E. H. (2003). Realworld illumination and the perception of surface reflectance properties. Journal of Vision, 3(5), 347-368.

Geisler-Moroder, D., \& Dür, A. (2010). A new Ward BRDF model with bounded albedo. Computer Graphics Forum, 29(4), 1391-1398.

Gelman, A., Goegebeur, Y., Tuerlinckx, F., \& Mechelen, I. V. (2000). Diagnostic checks for discrete data regression models using posterior predictive simulations. Journal of the Royal Statistical Society. Series $C$ (Applied Statistics), 49(2), 247-268.

Hoffman, M. D., \& Gelman, A. (2014). The No-U-Turn sam- pler: Adaptively setting path lengths in Hamiltonian Monte Carlo. Journal of Machine Learning Research, 15, 1593-1623.

Joh, A., Adolph, K., Campbell, M., \& Eppler, M. (2006). Why walkers slip: Shine is not a reliable cue for slippery ground. Perception \& Psychophysics, 68(3), 339352.

Karim, A. K. M. R., \& Kojima, H. (2010). The what and why of perceptual asymmetries in the visual domain. Advances in Cognitive Psychology, 6, 103-115.

Kingdom, F. A., \& Prins, N. (2010). Psychophysics: A practical introduction. London, England: Elsevier.

Kleiner, M., Brainard, D., \& Pelli, D. (2007). What's new in Psychtoolbox-3? Perception, 36, ECVP Abstract Supplement.

Knoblauch, K., \& Maloney, L. (2008). MLDS: Maximum Likelihood Difference Scaling in R. Journal of Statistical Software, 25(2), 1-26.

Kontsevich, L. L., \& Tyler, C. W. (1999). Bayesian adaptive estimation of psychometric slope and threshold. Vision Research, 39(16), 2729-2737.

Kruschke, J. K., \& Liddell, T. M. (2018). The Bayesian new statistics: Hypothesis testing, estimation, metaanalysis, and power analysis from a Bayesian perspective. Psychonomic Bulletin \& Review, 25, 178-206.

Kuss, M., Jäkel, F., \& Wichmann, F. A. (2005). Bayesian inference for psychometric functions. Journal of Vision, 5(5), 478-492.

Lee, M. D. (2018). Bayesian methods in cognitive modeling. In J. T. Wixted (Ed.), Stevens' handbook of experimental psychology and cognitive neuroscience (4th ed., Vol. 5, pp. 1-48). John Wiley \& Sons, Inc.

Lesch, M. F., Chang, W.-R., \& Chang, C.-C. (2008). Visually based perceptions of slipperiness: underlying cues, consistency and relationship to coefficient of friction. Ergonomics, 51(12), 1973-1983.

Liu, T., Heeger, D. J., \& Carrasco, M. (2006). Neural correlates of the visual vertical meridian asymmetry. Journal of Vision, 6(11), 1294-1306.

Makowski, D., Ben-Shachar, M. S., \& Lüdecke, D. (2019). bayestestR: Describing effects and their uncertainty, existence and significance within the Bayesian framework. Journal of Open Source Software, 4(40), 1541.

Maloney, L. T., \& Yang, J. N. (2003). Maximum likelihood difference scaling. Journal of Vision, 3(8), 573-585.

Marigold, D. S., \& Patla, A. E. (2008). Visual information from the lower visual field is important for walking across multi-surface terrain. Experimental Brain Research, 188(1), 23-31.

Marigold, D. S., Weerdesteyn, V., Patla, A. E., \& Duysens, J. (2007). Keep looking ahead? Re-direction of visual fixation does not always occur during an unpre- 

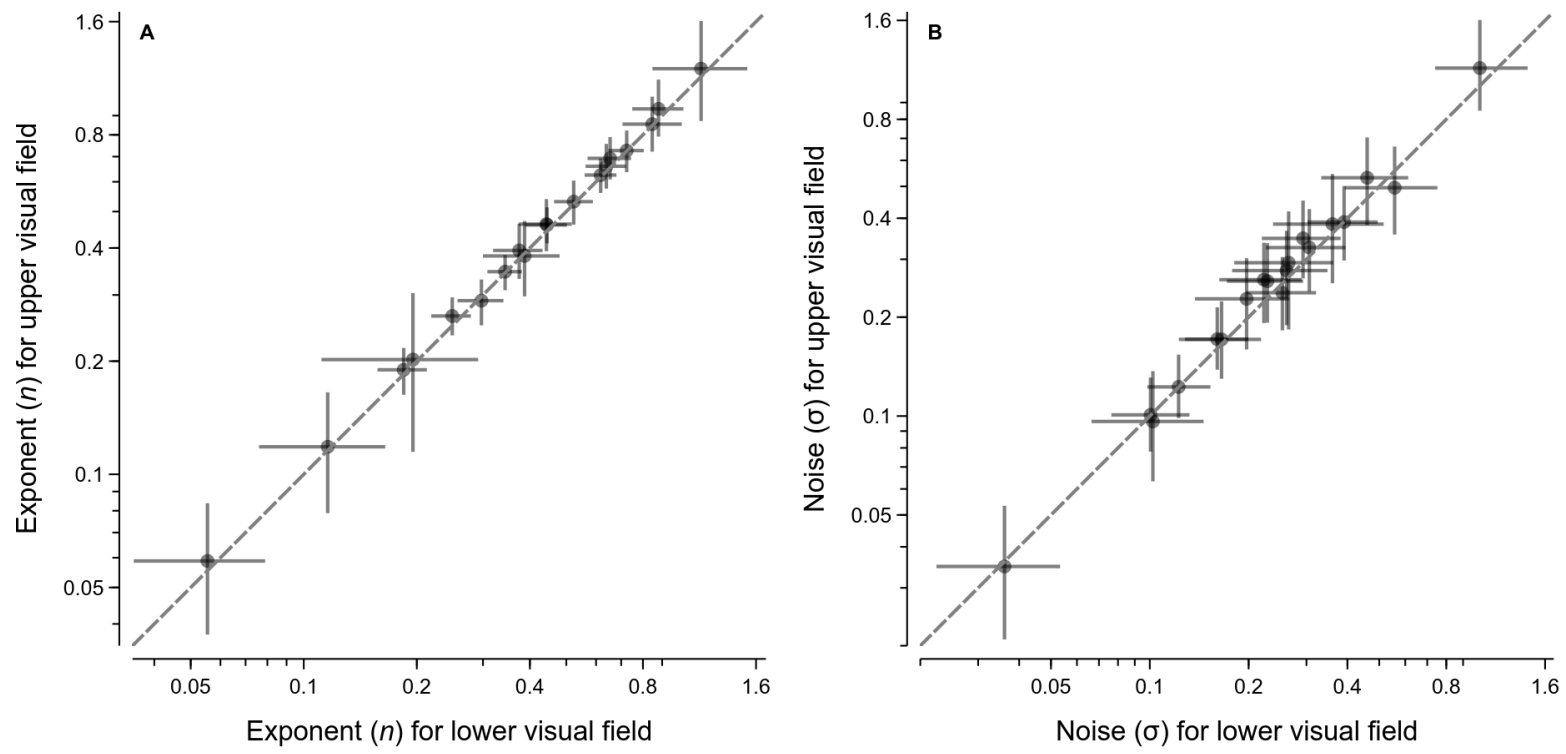

Figure 10. Participant-specific exponent (A) and noise (B) estimates. The horizontal and vertical axes show the parameter values (with log spacing) for where the stimuli were shown in the lower and upper visual fields, respectively. In each panel, each point shows the median (circles) and marginal 90\% credible intervals (lines) for the posterior pushforward distribution formed from the combination of the fixed effect estimates and the estimates of a given participant's random effect. The dashed line in each panel shows the line of unity.

dictable obstacle avoidance task. Experimental Brain Research, 176(1), 32-42.

Marlow, P., Kim, J., \& Anderson, B. (2012). The perception and misperception of specular surface reflectance. Current Biology, 22(20), 1909-1913.

Obein, G., Knoblauch, K., \& Viéot, F. (2004). Difference scaling of gloss: Nonlinearity, binocularity, and constancy. Journal of Vision, 4(9), 711-720.

Pellacini, F., Ferwerda, J. A., \& Greenberg, D. P. (2000). Toward a psychophysically-based light reflection model for image synthesis. In Proceedings of the 27th annual conference on computer graphics and interactive techniques (pp. 55-64).

Pelli, D. G. (1997). The VideoToolbox software for visual psychophysics: transforming numbers into movies. Spatial Vision, 10(4), 437-442.

Phillips, J. B., Ferwerda, J. A., \& Luka, S. (2009). Effects of image dynamic range on apparent surface gloss. In 17th color and imaging conference (Vol. 2009, pp. 193-197). Bellingham, WA: Society for Imaging Science and Technology.

Previc, F. H. (1990). Functional specialization in the lower and upper visual fields in humans: Its ecological origins and neurophysiological implications. Behavioral and Brain Sciences, 13, 519-542.

Prins, N., \& Kingdom, F. A. A. (2018). Applying the model- comparison approach to test specific research hypotheses in psychophysical research using the Palamedes toolbox. Frontiers in Psychology, 9(1250), 1-14.

Rijsdijk, J., Kroon, J., \& van der Wildt, G. (1980). Contrast sensitivity as a function of position on the retina. Vision Research, 20(3), 235-241.

Salvatier, J., Wiecki, T. V., \& Fonnesbeck, C. (2016). Probabilistic programming in Python using PyMC3. PeerJ Computer Science, 2(e55), 1-24.

Samadzadegan, S., Baar, T., Urban, P., Segovia, M. V. O., \& Blahová, J. (2015). Controlling colour-printed gloss by varnish-halftones. In M. V. O. Segovia, P. Urban, \& F. H. Imai (Eds.), Measuring, modeling, and reproducing material appearance 2015 (Vol. 9398, pp. 283 292). SPIE.

Sun, H.-C., Welchman, A. E., Chang, D. H., \& Di Luca, M. (2016). Look but don't touch: Visual cues to surface structure drive somatosensory cortex. NeuroImage, 128, 353-361.

Timmis, M. A., Bennett, S. J., \& Buckley, J. G. (2009). Visuomotor control of step descent: evidence of specialised role of the lower visual field. Experimental Brain Research, 195(2), 219-227.

van de Schoot, R., Depaoli, S., King, R., Kramer, B., Märtens, K., Tadesse, M. G., .. Yau, C. (2021). Bayesian statistics and modelling. Nature Reviews 

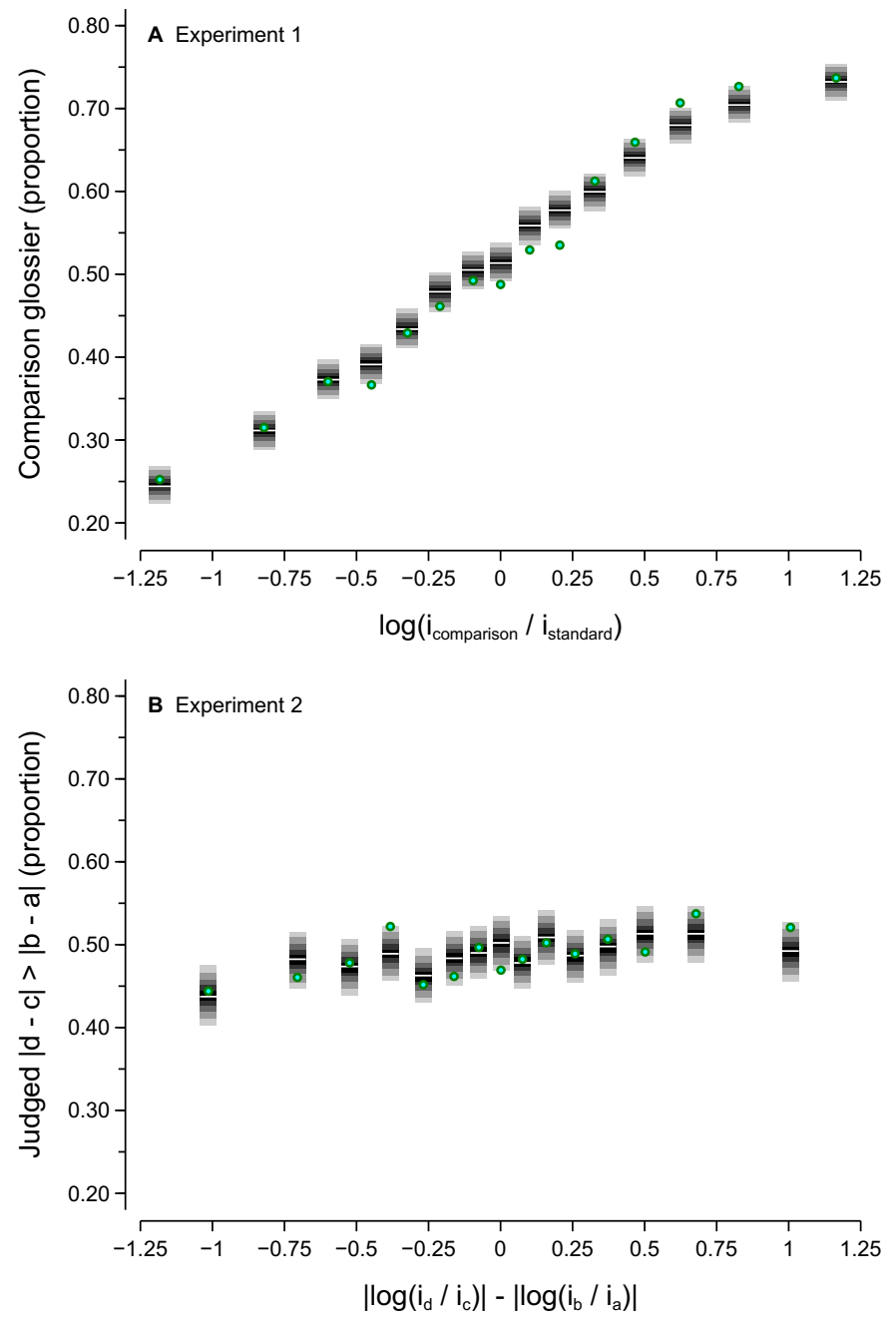

Figure 11. The relationship between the simulated illumination of objects within a trial and judgements of glossiness, aggregated over participants. Panel $\mathbf{A}$ is for Experiment 1 and shows the proportion of trials where the comparison object was judged as glossier than the standard object (vertical axis) as a function of the log of the ratio of comparison and standard object illumination (horizontal axis). Panel B is for Experiment 2 and shows the proportion of trials where the absolute difference in gloss between the $(c, d)$ stimulus pair was judged as greater than between the $(a, b)$ stimulus pair (vertical axis) as a function of the absolute differences of the log ratios of paired object illumination levels (horizontal axis). The figure conventions are as in Figure 3.
Methods Primers, 1(1), 1-26.

Wagenmakers, E.-J., Marsman, M., Jamil, T., Ly, A., Verhagen, J., Love, J., ... Morey, R. D. (2018). Bayesian inference for psychology. Part I: Theoretical advantages and practical ramifications. Psychonomic Bulletin \& Review, 25, 35-57.

Wallis, T. S. A., Dorr, M., \& Bex, P. J. (2015). Sensitivity to gaze-contingent contrast increments in naturalistic movies: An exploratory report and model comparison. Journal of Vision, 15(8), 1-33.

Walter, B. (2005). Notes on the Ward BRDF (Tech. Rep. No. PCG-05-06). Program of Computer Graphics, Cornell University.

Ward, G. J. (1992). Measuring and modeling anisotropic reflection. In Proceedings of the 19th annual conference on computer graphics and interactive techniques (p. 265-272). New York, NY, USA: Association for Computing Machinery.

Wendt, G., Faul, F., Ekroll, V., \& Mausfeld, R. (2010). Disparity, motion, and color information improve gloss constancy performance. Journal of Vision, 10(9), 117 . 